\title{
Distinguishing numbers for graphs and groups
}

\author{
Julianna Tymoczko* \\ Department of Mathematics \\ University of Michigan, Ann Arbor, MI 48109 \\ tymoczko@umich.edu
}

Submitted: Jan 30, 2004; Accepted: Aug 25, 2004; Published: Sep 16, 2004

2000 MR Subject Classification 05C15, 05C25, 20D60

\begin{abstract}
A graph $G$ is distinguished if its vertices are labelled by a map $\phi: V(G) \longrightarrow$ $\{1,2, \ldots, k\}$ so that no non-trivial graph automorphism preserves $\phi$. The distinguishing number of $G$ is the minimum number $k$ necessary for $\phi$ to distinguish the graph. It measures the symmetry of the graph.

We extend these definitions to an arbitrary group action of $\Gamma$ on a set $X$. A labelling $\phi: X \longrightarrow\{1,2, \ldots, k\}$ is distinguishing if no element of $\Gamma$ preserves $\phi$ except those which fix each element of $X$. The distinguishing number of the group action on $X$ is the minimum $k$ needed for $\phi$ to distinguish the group action. We show that distinguishing group actions is a more general problem than distinguishing graphs.

We completely characterize actions of $S_{n}$ on a set with distinguishing number $n$, answering an open question of Albertson and Collins.
\end{abstract}

\section{Introduction}

Consider the following dilemma of the considerate roommate. Returning home late at night, she would like to unlock her door without disturbing her roommates either by turning on a light or by repeatedly trying incorrect keys in the lock. One solution is to put different handles on her keys so that no matter how her keychain is oriented she can identify each key simply by its shape and its order on the chain. This leads to a natural question: what is the minimum number of handles needed to tell her keys apart?

Motivated by this puzzle, Albertson and Collins defined a distinguished graph to be one whose vertices are labelled by a function $\phi: V(G) \longrightarrow\{1, \ldots, k\}$ so that no non-trivial

*Part of this research was done at the Summer Research Program at the University of Minnesota, Duluth sponsored by the National Science Foundation (DMS-9225045) and the National Security Agency (MDA904-91-H-0036). 
graph automorphism preserves the labelling [AC1]. From this perspective, the standard keychain corresponds to a cyclic graph on which each vertex corresponds to a key. With their definition, Albertson and Collins extended the puzzle to ask what happens when a keychain is shaped unusually, for instance like a barbell or like the edges of a cube.

Albertson and Collins defined the distinguishing number of a graph to be the minimum number of labels $k$ necessary to distinguish the graph. In the case of the cyclic graph, they reproved the classical result that the cyclic graphs $C_{3}, C_{4}$, and $C_{5}$ require three labels but that the other cyclic graphs only need two. In other words, either two or three handles are needed to tell keys apart by feel, depending on the number of keys on the chain. Figure 1 demonstrates an upper bound for the distinguishing numbers of $C_{5}$ and $C_{6}$. (The number inside the vertex $v$ is the labelling $\phi(v)$.) The reader can enumerate the possibilities to see that $C_{5}$ cannot be distinguished with fewer than three labels.
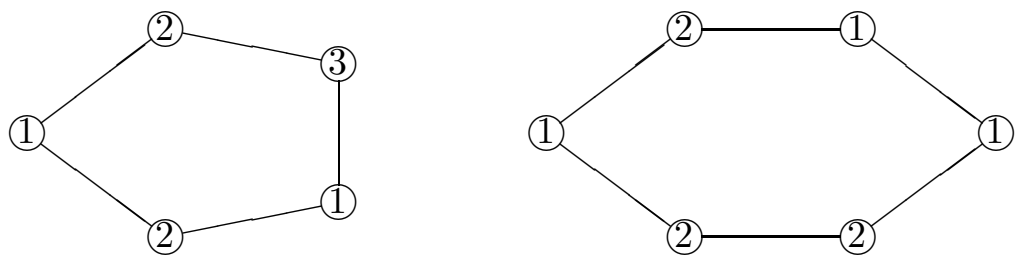

Figure 1: Minimally distinguished cyclic graphs

Graphs with the same automorphism group can nonetheless have different distinguishing numbers. Instead of asking what the distinguishing number of a fixed graph is, we may ask which distinguishing numbers are associated to a fixed group. In other words, given a group $\Gamma$ we ask for the set

$$
D_{\Gamma}=\{k: k=D(G) \text { for a graph } G \text { with } \operatorname{Aut}(G)=\Gamma\} .
$$

For instance, the wreath product $S_{2}\left[S_{3}\right]$ is the automorphism group of the graph consisting of two disjoint copies of the complete graph $K_{3}$. Figure 2 shows this and two other graphs with the same automorphism group and demonstrates that all three have different distinguishing numbers. This example disproves a conjecture in [AC2] that for no group $\Gamma$ is $D_{\Gamma}=\{2,3,4\}$. In fact, any graph with automorphism group $S_{2}\left[S_{3}\right]$ must have distinguishing number at least 2 by definition and no more than 4 by Theorem 2.14 , and all three possibilities can be realized.
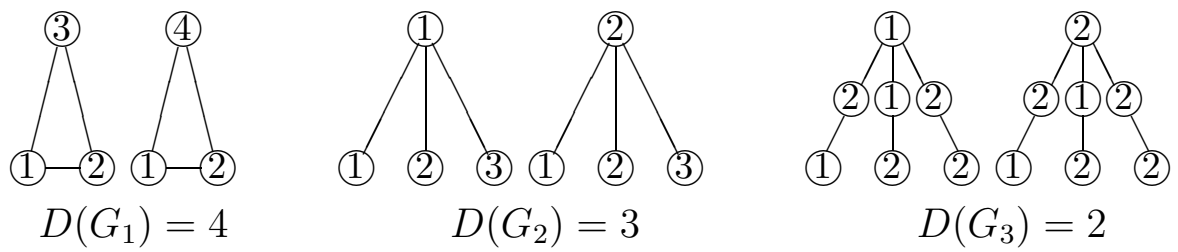

Figure 2: Three graphs with automorphism group $S_{2}\left[S_{3}\right]$

In this paper we extend the notion of distinguishing numbers to an arbitrary group action on a set. This definition is quite natural since distinguishing graphs often in- 
volves studying the action of the automorphism group on a single vertex orbit, in effect considering a more general group action on a particular set of vertices.

Section 2 discusses distinguishing numbers of general group actions in more detail. The distinguishing numbers of some common group actions are computed, including translations as well as conjugations by $S_{n}$ on various sets. This section also gives an orbit-by-orbit construction of a distinguishing labelling for arbitrary group actions, which generalizes an analogous construction from the theory of distinguishing graphs. Theorem 2.15 completely characterizes group actions on a set which have distinguishing number $n$ when the group has order $n$ !, proving in this case that all of the group orbits have size one, except for one orbit with $n$ elements upon which the group acts as all possible permutations.

Section 3 shows that distinguishing numbers of graphs and general group actions are substantively different. This section contains a proof that a faithful $S_{4}$-action on a set has distinguishing number 2,3 , or 4 by demonstrating $S_{4}$-actions with each of these distinguishing numbers. By contrast, Albertson and Collins showed that no graph has automorphism group $S_{4}$ and distinguishing number 3 in [AC1].

Section 4 uses group actions to compute distinguishing numbers of graphs. Theorem 4.1 proves that the distinguishing number of a tree is bounded by its maximum degree and that this bound is sharp. This is similar to work in [Ch, 2.2.4 and 2.2.5], which uses a different approach than that taken in this paper. This section also contains Theorem 4.2, which uses Theorem 2.15 to prove a conjecture of [AC1] that any graph with automorphism group $S_{n}$ and distinguishing number $n$ is either $K_{n}$ or $\overline{K_{n}}$ as well as any number of 1-orbits.

The author is very grateful to Daniel Isaksen for suggesting the study of general group actions, to Karen Collins and Michael Albertson for many helpful conversations, and to the referees for very useful suggestions.

\section{Distinguishing group actions}

Any group action on a set can be distinguished, not just that of the automorphism group on a graph. In fact these more general group actions arise frequently, for instance as the action of the automorphism group of a graph on one of its vertex orbits. This highlights the main algebraic difference between distinguishing groups and distinguishing graphs: many groups do not act faithfully while the automorphism group of a graph always has trivial stabilizer.

In this section we define the distinguishing number of a group action and compute it for some examples, including translation and conjugation actions. We demonstrate two different ways to construct a labelling orbit-by-orbit and show how these can be used to bound the distinguishing number by $k$ when the group has order at most $k$ !. The main steps are Theorems 2.9 and 2.10, which generalize an unpublished proof of Albertson, Collins, and Kleitman [Co]. In Theorem 2.15 we prove a more general version of a conjecture by Albertson and Collins that characterizes completely those sets acted on by a group of order $n$ ! with distinguishing number $n$.

Let $\Gamma$ be a group which acts on the set $X$. If $g$ is an element of $\Gamma$ and $x$ is in $X$ then denote the action of $g$ on $x$ by $g . x$. Write $\Gamma . x$ for the orbit containing $x$. Recall that the 
stabilizer of the subset $Y \subseteq X$ is defined to be

$$
\operatorname{Stab}_{\Gamma}(Y)=\{g \in \Gamma: g \cdot y=y \text { for all } y \in Y\} .
$$

We sometimes omit the subscript and write $\operatorname{Stab}(Y)$. We also use $\langle g\rangle$ to denote the subgroup of $\Gamma$ generated by $g$. Assume all groups and sets are finite.

A labelling of $X$ is a map $\phi: X \longrightarrow\{1,2, \ldots, k\}$. We say that $\phi$ is a $k$-distinguishing labelling if the only group elements that preserve the labelling are in $\operatorname{Stab}(X)$. Equivalently, the map $\phi$ is a $k$-distinguishing labelling if $\{g: \phi \circ g=\phi\}=\operatorname{Stab}(X)$. The distinguishing number $D_{\Gamma}(X)$ of the set $X$ with a given group action of $\Gamma$ is the minimum $k$ for which there is a $k$-distinguishing labelling.

For example, consider what happens when $S_{3}$ acts by conjugation on itself. There are three orbits under this action, namely the three conjugacy classes of $S_{3}$. Figure 3 shows these orbits with lines between elements of $S_{3}$ if the two elements are conjugate. The stabilizer of the transposition (12) is the group generated by (12). Similarly the stabilizer of (123) is the group generated by (123). Consequently the labelling given by

$$
\begin{aligned}
& \phi(12)=\phi(123)=2, \text { and } \\
& \phi(x)=1 \text { otherwise }
\end{aligned}
$$

is a 2-distinguishing labelling of $S_{3}$ under the conjugation action. This labelling is shown in Figure 3. Theorem 2.5 generalizes this example to show that whenever $S_{n}$ acts on itself by conjugation its distinguishing number is 2 .

id

(1)

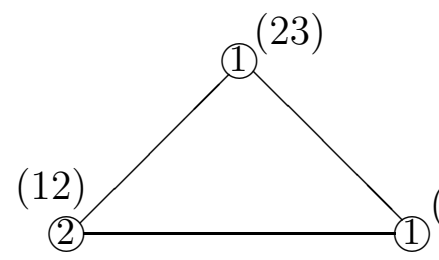

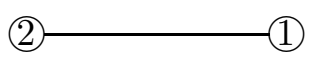

Figure 3: $S_{3}$ acts on itself by conjugation

The first proposition follows immediately from the definitions.

Proposition 2.1. The group $\Gamma$ acts on the set $X$ by fixing each element if and only if $D_{\Gamma}(X)=1$.

The next proposition computes the distinguishing number when $\Gamma$ acts on itself by translation.

Proposition 2.2. If $\Gamma$ acts on itself by translation then $D_{\Gamma}(\Gamma)=2$.

Proof. Fix $h_{0}$ in $\Gamma$ and define the labelling

$$
\phi(h)= \begin{cases}2 & \text { if } h=h_{0}, \text { and } \\ 1 & \text { otherwise. }\end{cases}
$$

If $g$ preserves the labelling then $\phi\left(g \cdot h_{0}\right)=\phi\left(h_{0}\right)=2$. This implies that $g \cdot h_{0}=h_{0}$. Since $g \cdot h_{0}=g h_{0}$ the element $g$ must be the identity. 
The next lemma is a basic tool to recursively construct distinguishing labellings.

Lemma 2.3. Fix an orbit $O$ under the action of $\Gamma$ on $X$. Let $\phi_{1}$ be a $\left(k_{1}\right)$-distinguishing labelling of $O$ under the action of $\Gamma$ and let $\phi_{2}$ be a $\left(k_{2}\right)$-distinguishing labelling of $X \backslash O$ under the action of $\Gamma$. The labelling $\phi$ defined by $\left.\phi\right|_{O}=\phi_{1}$ and $\left.\phi\right|_{X \backslash O}=\phi_{2}$ is a $\max \left\{k_{1}, k_{2}\right\}$ distinguishing of $X$ under the action of $\Gamma$.

Proof. If $g$ preserves the labelling $\phi$ then $g$ preserves both $\phi_{O}$ and $\phi_{X \backslash O}$. Consequently $g$ is in the subgroup $\operatorname{Stab}(O) \cap \operatorname{Stab}(X \backslash O)$ and so $g$ is in $\operatorname{Stab}(X)$.

This gives a numerical condition for 2-distinguishability.

Corollary 2.4. Suppose $\Gamma$ acts on $X$ and $O_{1}$ and $O_{2}$ are two orbits under this action. If $|\Gamma| /\left|O_{1}\right|$ is relatively prime to $|\Gamma| /\left|O_{2}\right|$ then $D_{\Gamma}(X)=2$.

Proof. Choose $x_{1} \in O_{1}$ and $x_{2} \in O_{2}$. Define a labelling $\phi: X \longrightarrow\{1,2\}$ by setting $\phi\left(x_{1}\right)=\phi\left(x_{2}\right)=2$ and $\phi(x)=1$ for all other $x$. If $g$ preserves $\phi$ then it satisfies

$$
\phi\left(g . x_{1}\right)=2=\phi\left(g \cdot x_{2}\right)
$$

Since $g . x_{i} \in O_{i}$ and $x_{i}$ is the only element of $O_{i}$ labelled by 2, the action of $g$ must fix each of $x_{1}$ and $x_{2}$. In other words $g$ is in $\operatorname{Stab}\left(x_{1}\right) \cap \operatorname{Stab}\left(x_{2}\right)$. The cardinality of these stabilizer subgroups is given by $\left|\operatorname{Stab}\left(x_{i}\right)\right|=|\Gamma| /\left|O_{i}\right|$ as shown in [L, 1.5.1 and 1.2.2]. These cardinalities are relatively prime by hypothesis so the intersection of the stabilizers is the identity. Consequently $\phi$ is a 2-distinguishing labelling.

This result can be used to distinguish the action of $S_{n}$ on itself by conjugation since relatively prime orbits can be constructed in that case.

Theorem 2.5. Let $X$ be the set of permutations $S_{n}$ with the group action of $S_{n}$ upon $X$ given by conjugation. Then $D_{S_{n}}\left(S_{n}\right)=2$.

Proof. The orbits of $S_{n}$ acting on itself by conjugation are the conjugacy classes of $S_{n}$ and are characterized by cycle type, that is by partitions of $n$ (see [FH, 2.3 page 18]).

One orbit corresponds to the cycle type of the permutation $(12 \cdots n)$. The number of $n$-cycles is $(n-1)$ ! since each $n$-cycle $\sigma$ is determined uniquely by a sequence $\left(\sigma^{1}(1), \sigma^{2}(1), \ldots, \sigma^{n}(1)\right)$ which has $\sigma^{n}(1)=1$ and which permutes the other $n-1$ elements. Consequently the stabilizer of $(12 \cdots n)$ has size $\frac{n !}{(n-1) !}=n$.

Another orbit corresponds to the cycle type of $(1)(2 \cdots n)$, which fixes one element and has an $n-1$ cycle. This orbit has size $n(n-2)$ ! since there are $n$ choices for the fixed element and $(n-2)$ ! ways to choose an $n-1$ cycle. Consequently the stabilizer of $(1)(2 \cdots n)$ has size $\frac{n !}{n(n-2) !}=n-1$.

Since $n$ and $n-1$ are relatively prime, this group action is 2-distinguishable by Corollary 2.4 .

The next lemma can be used to construct distinguishing labellings by looking at orbits under stabilizer subgroups. 
Lemma 2.6. Fix an action of $\Gamma$ on $X$ and let $X^{\prime}$ be a subset of $X$ with $\Gamma . x \neq \Gamma$.y whenever $x$ and $y$ are two distinct elements in $X^{\prime}$. If $\phi_{1}$ is a $(k-1)$-distinguishing labelling of $X \backslash X^{\prime}$ under the action of the subgroup $\operatorname{Stab}\left(X^{\prime}\right)$ then the map

$$
\phi(u)= \begin{cases}\phi_{1}(u) & \text { if } u \notin X^{\prime}, \text { and } \\ k & \text { if } u \in X^{\prime}\end{cases}
$$

is a $k$-distinguishing labelling of $X$ under the action of $\Gamma$.

Proof. If $g$ preserves the labelling $\phi$ then both $\phi_{1} \circ g=\phi_{1}$ and $\phi(g . x)=k$ for each $x$ in $X^{\prime}$. No two elements in $X^{\prime}$ lie in the same $\Gamma$-orbit and so $g \cdot x=x$ for each $x$ in $X^{\prime}$. Consequently $g$ is in $\operatorname{Stab}\left(X^{\prime}\right)$. Moreover the labelling $\phi_{1}$ distinguishes $X \backslash X^{\prime}$ under $\operatorname{Stab}\left(X^{\prime}\right)$ and so $g$ must also be in $\operatorname{Stab}\left(X \backslash X^{\prime}\right)$. Consequently $g$ is in $\operatorname{Stab}(X)$.

We use this lemma to construct a distinguishing labelling for the action of $\Gamma$ on $X$ using the following recursive algorithm.

\section{Construction 2.7.}

1. Initialize $i=1$ and set $\phi(x)=1$ for all $x$ in $X$. Let $\Gamma_{1}=\Gamma$ and $X_{1}=X$.

2. While $\Gamma_{i} \neq \operatorname{Stab}\left(X_{i}\right)$ do

(a) Choose a subset $X_{i+1}^{\prime}$ of $X_{i}$ that contains a unique element from each nontrivial $\Gamma_{i}$-orbit in $X_{i}$, namely so that the intersection $\left|X_{i+1}^{\prime} \cap \Gamma_{i} \cdot x\right|=1$ for each $x$ in $X_{i}$ such that $\Gamma_{i} . x$ has at least two elements.

(b) Label the elements of $X_{i+1}^{\prime}$ with $i+1$, so $\phi(x)=i+1$ for each $x$ in $X_{i+1}^{\prime}$.

(c) Let $X_{i+1}=X_{i} \backslash X_{i+1}^{\prime}$ and let $\Gamma_{i+1}=\operatorname{Stab}_{\Gamma_{i}}\left(X_{i+1}^{\prime}\right)$.

(d) Increment $i$ by 1 .

Figure 4 gives an example of how this works when the set $X$ is the set of vertices of the given graph and the group $\Gamma$ consists of all graph automorphisms. In this case the algorithm terminates after the loop is iterated three times. Comparing the outcome to Figure 2 we observe that this algorithm need not give a minimal distinguishing labelling.

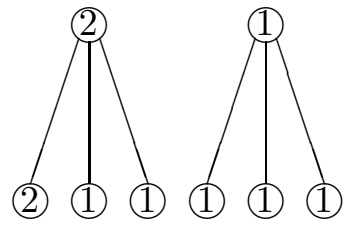

First Iteration

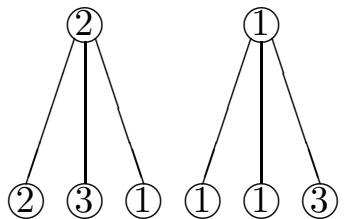

Second Iteration

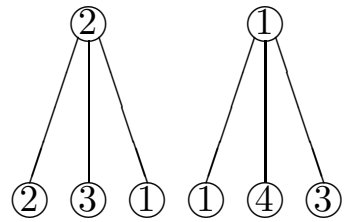

Third Iteration

Figure 4: Constructing a 4-distinguishing labelling

The following uses Lemma 2.6 to confirm that this produces a distinguishing labelling. 
Proposition 2.8. Construction 2.7 terminates after $k-1$ iterations and produces a $k$ distinguishing labelling $\phi$ of $X$, for some finite $k$.

Proof. Since $X_{i} \subsetneq X_{i-1}$ the algorithm terminates after at most $|X|$ iterations. We induct on $k$. When $\Gamma=\operatorname{Stab}(X)$ the algorithm uses no iterations and the map $\phi$ is trivially a 1distinguishing labelling. Assume that any labelling produced when the algorithm requires $k-2$ iterations is a $k-1$-distinguishing labelling.

Suppose $\phi$ is produced when the algorithm uses $k-1$ iterations. By construction, the element $x$ in $X$ is labelled $\phi(x)=i>1$ if and only if $x$ is in $X_{i}^{\prime}$. Thus $\phi$ is a $k$-labelling.

Furthermore, the set $X_{2}^{\prime}$ has no more than one element from each $\Gamma$-orbit, by construction. The restriction $\left.\phi\right|_{X_{2}}$ is a $k$-1-distinguishing labelling under the action of $\Gamma_{2}$, by the inductive hypothesis. By Lemma 2.6 , the map $\phi$ is a $k$-distinguishing labelling.

The construction in fact guarantees that there is a set of $k$ nested orbits under successively smaller stabilizer subgroups.

Theorem 2.9. Fix a k-distinguishing labelling $\phi$, groups $\left\{\Gamma_{i}\right\}$, and sets $\left\{X_{i}\right\}$ produced by an implementation of Construction 2.7. There exists a subset $\left\{y_{1}, \ldots, y_{k}\right\}$ in $X$ such that

1. $\phi\left(y_{i}\right)=i$ for each $i$,

2. $y_{i+1}$ is in $\Gamma_{i-1} . y_{i}$ for each $i$ from 2 to $k-1$, and $y_{1}$ is in $\Gamma_{k-1} \cdot y_{k}$.

Proof. The algorithm uses $k-1$ iterations so the groups satisfy $\Gamma_{k-1} \neq \operatorname{Stab}\left(X_{k-1}\right)$ and $\Gamma_{k}=\operatorname{Stab}\left(X_{k}\right)$. This means there is an element $y_{1}$ in $X_{k}$ whose orbit $\Gamma_{k-1} \cdot y_{1}$ has at least two elements. Note that $\phi\left(y_{1}\right)=1$ by construction. Also by construction, the orbit $\Gamma_{k-1} \cdot y_{1}$ intersects $X_{k}^{\prime}$ in a unique element $y_{k}$, and $y_{k}$ is labelled $\phi\left(y_{k}\right)=k$.

Assume that $\left\{y_{i}, y_{i+1}, \ldots, y_{k}, y_{1}\right\}$ have been chosen to satisfy the hypotheses. In particular, the orbit $\Gamma_{i-1} \cdot y_{i}$ has at least two elements. Since $\Gamma_{i-2} \supset \Gamma_{i-1}$ the orbit $\Gamma_{i-2} \cdot y_{i}$ also has at least two elements and so this orbit intersects $X_{i-1}^{\prime}$ in a unique element $y_{i-1}$. By construction $\phi\left(y_{i-1}\right)=i-1$ and $\Gamma_{i-2} \cdot y_{i-1}=\Gamma_{i-2} . y_{i}$. Induction completes the proof.

Together with the coset formula for group orders, the construction guarantees a lower bound on the size of orbits under successively smaller stabilizer subgroups. This bound generalizes the main point of an earlier construction of Albertson, Collins, and Kleitman in $[\mathrm{AC} 2]$ whose proof is unpublished $[\mathrm{Co}]$.

Theorem 2.10. Fix a $k$-distinguishing labelling $\phi$, groups $\left\{\Gamma_{i}\right\}$, and sets $\left\{X_{i}\right\}$ produced by an implementation of Construction 2.7. If $\left\{y_{1}, \ldots, y_{j}\right\}$ is a subset of $X$ such that

1. $\phi\left(y_{i}\right)=i$ for each $i$,

2. $y_{i+1}$ is in $\Gamma_{i-1} \cdot y_{i}$ for each $i$ from 2 to $j-1$, and $y_{1}$ is in $\Gamma_{j-1} \cdot y_{j}$,

then $|\Gamma| \geq\left|\Gamma_{1} \cdot y_{2}\right|\left|\Gamma_{2} \cdot y_{3}\right| \cdots\left|\Gamma_{j-1} \cdot y_{j}\right|\left|\Gamma_{j} \cdot y_{1}\right|\left|\operatorname{Stab}_{\Gamma_{j}}\left(y_{1}\right)\right|$. 
Proof. Recall that whenever a group $\Gamma$ acts on a set $X$ and $y$ is in $X$, the orders satisfy

$$
|\Gamma|=|\Gamma \cdot y| \cdot\left|\operatorname{Stab}_{\Gamma}(y)\right|
$$

(see [L, 1.5.1 and 1.2.2]). Observe that for each $i \neq 1$ the group $\operatorname{Stab}_{\Gamma_{i-1}}\left(y_{i}\right) \supseteq \Gamma_{i}$ since $\Gamma_{i}=\bigcap_{y \in X_{i}^{\prime}} \operatorname{Stab}_{\Gamma_{i-1}}(y)$ by definition. Together, these identities give the following:

$$
\begin{aligned}
|\Gamma| & =\left|\Gamma \cdot y_{2}\right|\left|\operatorname{Stab}_{\Gamma}\left(y_{2}\right)\right| \\
& \geq\left|\Gamma \cdot y_{2}\right|\left|\Gamma_{2}\right| \\
& =\left|\Gamma \cdot y_{2}\right|\left|\Gamma_{2} \cdot y_{3}\right|\left|\operatorname{Stab}_{\Gamma_{2}}\left(y_{3}\right)\right| \\
& \cdots \\
& =\left|\Gamma \cdot y_{2}\right|\left|\Gamma_{2} \cdot y_{3}\right| \cdots\left|\Gamma_{j-1} \cdot y_{j}\right|\left|\Gamma_{j} \cdot y_{1}\right|\left|\operatorname{Stab}_{\Gamma_{j}}\left(y_{1}\right)\right| .
\end{aligned}
$$

Since $\Gamma=\Gamma_{1}$ this proves the claim.

The next corollary uses this result and a lower bound for each $\left|\Gamma_{i-1} . y_{i}\right|$ to bound $|\Gamma|$.

Corollary 2.11. Fix a k-distinguishing labelling $\phi$, groups $\left\{\Gamma_{i}\right\}$, and sets $\left\{X_{i}\right\}$ produced by an implementation of Construction 2.7. If $|\Gamma| \leq m$ ! and the subset $\left\{y_{1}, \ldots, y_{j}\right\}$ satisfies

1. $\phi\left(y_{i}\right)=i$ for each $i$,

2. $y_{i+1}$ is in $\Gamma_{i-1} . y_{i}$ for each $i$ from 2 to $j-1$, and $y_{1}$ is in $\Gamma_{j-1} \cdot y_{j}$,

then $j \leq m$ and $\left|\Gamma_{i-1} . y_{i}\right| \geq j-i+2$ for each $i$ from 2 to $j$.

Proof. We first show that the set $\Gamma_{i-1} . y_{i}$ contains $\left\{y_{i}, y_{i+1}, \ldots, y_{j}, y_{1}\right\}$ for each $i$. Indeed, the orbit $\Gamma_{i-1} \cdot y_{i}=\Gamma_{i-1} . y_{i+1}$ by definition. Since the subgroup $\Gamma_{i-1} \supset \Gamma_{i}$ it follows that $\Gamma_{i-1} \cdot y_{i+1} \supset \Gamma_{i} \cdot y_{i+1}$. (The containment $\Gamma_{i-1} \cdot y_{i+1} \supsetneq \Gamma_{i} \cdot y_{i+1}$ is proper because $\Gamma_{i}$ fixes $y_{i}$.) Consequently, the claim need only hold for $\Gamma_{j-1} . y_{j}$, which it does by hypothesis. Thus, each orbit $\Gamma_{i-1} \cdot y_{i}$ contains at least $j-i+2$ elements.

By Theorem 2.10

$$
|\Gamma| \geq\left|\Gamma_{1} \cdot y_{2}\right|\left|\Gamma_{2} \cdot y_{3}\right| \cdots\left|\Gamma_{j-1} \cdot y_{j}\right|\left|\Gamma_{j} \cdot y_{1}\right|\left|\operatorname{Stab}_{\Gamma_{j}}\left(y_{1}\right)\right|
$$

so $|\Gamma| \geq j$ !. By hypothesis $m ! \geq|\Gamma|$ so $j$ is at most $m$.

This corollary relates $|\Gamma|$ to the number of iterations of Construction 2.7 and thus to the distinguishing number.

Corollary 2.12. If $|\Gamma|$ is at most $k$ ! then $D_{\Gamma}(X)$ is at most $k$.

Proof. Let $\phi$ be an $j$-distinguishing labelling produced by Construction 2.7. By Theorem 2.9 there exist $j$ elements satisfying the conditions of Corollary 2.11 so $j$ is at most $k$.

This construction actually distinguishes each orbit of a group action separately.

Corollary 2.13. Suppose $|\Gamma| \leq k$ !. If $\Gamma$ acts on $X$ and $O$ is any orbit of this action then $O$ can be distinguished under the action of $\Gamma$ with at most $k$ labels. 
Proof. Apply Corollary 2.12 to the action of $\Gamma$ on $O$.

The next result was originally formulated by Albertson, Collins, and Kleitman for graphs.

Corollary 2.14. (Albertson, Collins, and Kleitman) A graph $G$ with $|\operatorname{Aut}(G)| \leq k$ ! has distinguishing number $D(G) \leq k$.

Proof. Apply Corollary 2.12 to the action of the automorphism group of $G$ on the set of vertices of the graph $G$.

The following theorem completely characterizes group actions for which $|\Gamma|=n$ ! and the distinguishing number is $n$. It generalizes a conjecture of Albertson and Collins for graphs that is proven in Theorem 4.2.

The proof counts cardinalities to show that the set guaranteed by Theorem 2.9 must in fact consist of $n$ elements with an action of all $n$ ! permutations. It then demonstrates that any other non-trivial orbit would decrease the distinguishing number.

Note that this result is stronger than the analogous statement for graphs given in Theorem 4.2, because the edges in a graph constrain the way that the automorphism group can act. General group actions do not have this added structure.

Theorem 2.15. If $|\Gamma|=n$ ! and $\Gamma$ acts on $X$ with distinguishing number $n$ then there is an orbit $\Gamma . x$ in $X$ with $n$ elements upon which $\Gamma$ acts as the set of all possible permutations. The rest of the orbits in $X$ have size 1 .

Proof. If the distinguishing number of $\Gamma$ on $X$ is $n$ then by Lemma 2.3 there exists at least one orbit $\Gamma . x$ for which $D_{\Gamma}(\Gamma . x)$ is at least $n$. In particular the map $\phi$ given by implementing Construction 2.7 for the action of $\Gamma$ on $\Gamma . x$ is an $n$-distinguishing labelling. (Corollary 2.13 shows that $\phi$ is at most $n$-distinguishing. If $\phi$ used fewer than $n$ labels then $D_{\Gamma}(\Gamma . x)$ would be less than $\left.n.\right)$

We show first that $\Gamma . x$ consists of $n$ elements. By Theorem 2.9 we can find $\left\{y_{1}, \ldots, y_{n}\right\}$ in $\Gamma . x$ satisfying both $y_{i+1} \in \Gamma_{i-1} . y_{i}$ and $\phi\left(y_{i}\right)=i$. By Theorem 2.10 the inequality

$$
|\Gamma| \geq\left|\Gamma_{1} \cdot y_{2}\right|\left|\Gamma_{2} \cdot y_{3}\right| \cdots\left|\Gamma_{n-1} \cdot y_{n}\right|\left|\Gamma_{n} \cdot y_{1}\right|
$$

holds. Corollary 2.11 proved that $\Gamma_{i-1} . y_{i} \supseteq\left\{y_{i}, y_{i+1}, \ldots, y_{n}, y_{1}\right\}$ and so $|\Gamma| \geq n$ !. Because $|\Gamma|=n$ ! each orbit $\Gamma_{i-1} . y_{i}=\left\{y_{i}, y_{i+1}, \ldots, y_{n}, y_{1}\right\}$ must have exactly $n-i+2$ elements, and $\Gamma_{n} . y_{1}=\left\{y_{1}\right\}$. In particular note that $\Gamma_{1} \cdot y_{2}=\Gamma \cdot x=\left\{y_{1}, \ldots, y_{n}\right\}$.

We now show that $\Gamma$ acts on this orbit by all possible permutations. To begin we prove that $\Gamma_{i}=\operatorname{Stab}_{\Gamma}\left(y_{2}, \ldots, y_{i-1}\right)$ and that $\left|\Gamma_{i}\right|=(n-i+1)$ !. This is true by hypothesis when $i$ is one. Assume the claim holds for $\Gamma_{i-1}$. By the coset formula,

$$
\left|\Gamma_{i-1}\right|=\left|\Gamma_{i-1} \cdot y_{i}\right| \cdot\left|\operatorname{Stab}_{\Gamma_{i-1}}\left(y_{i}\right)\right| \text {. }
$$

Since $\left|\Gamma_{i-1} . y_{i}\right|=n-i+2$ this implies that $\left|\operatorname{Stab}_{\Gamma_{i-1}}\left(y_{i}\right)\right|=(n-i+1)$ !. By definition $\Gamma_{i} \subseteq \operatorname{Stab}_{\Gamma_{i-1}}\left(y_{i}\right)$. When Construction 2.7 is used for the action of $\Gamma_{i}$ on $\Gamma_{i} \cdot y_{i+1}$, the 
algorithm terminates after $n-i$ iterations. Theorem 2.10 shows that $\left|\Gamma_{i}\right| \geq(n-i+1)$ ! and so in fact $\Gamma_{i}=\operatorname{Stab}_{\Gamma_{i-1}}\left(y_{i}\right)$. By induction $\operatorname{Stab}_{\Gamma_{i-1}}\left(y_{i}\right)=\operatorname{Stab}_{\Gamma}\left(y_{2}, \ldots, y_{i}\right)$.

If $g$ and $h$ in $\Gamma$ act the same on the orbit $\Gamma . x$ then $g^{-1} h$ must be in $\operatorname{Stab}_{\Gamma}\left(y_{1}, \ldots, y_{n}\right)$. Since $\operatorname{Stab}_{\Gamma}\left(y_{1}, \ldots, y_{n}\right)$ has only one element this means $g^{-1} h$ is the identity. In other words, each of the $n$ ! elements in $\Gamma$ acts differently upon $\Gamma \cdot y_{1}=\left\{y_{1}, \ldots, y_{n}\right\}$. Since each element of $\Gamma$ permutes $\left\{y_{1}, \ldots, y_{n}\right\}$ the group acts as all possible permutations upon this $n$-element orbit.

Finally we confirm that every orbit other than $\Gamma . x$ is a 1-orbit. Suppose $O$ is any orbit other than $\Gamma . x$ and fix $x^{\prime}$ in $O$. Define a labelling of $O$ by

$$
\phi_{1}(y)= \begin{cases}2 & \text { if } y=x^{\prime}, \text { and } \\ 1 & \text { otherwise }\end{cases}
$$

The group elements that preserve this labelling are precisely those of $\operatorname{Stab}_{\Gamma}\left(x^{\prime}\right)$. Let $\phi_{2}$ be a $k$-distinguishing labelling of $X \backslash O$ under the action of $\operatorname{Stab}_{\Gamma}\left(x^{\prime}\right)$. If $g$ preserves $\phi_{2}$ then $g$ is in $\operatorname{Stab}_{\Gamma}(X \backslash O) \cap \operatorname{Stab}_{\Gamma}\left(x^{\prime}\right)=\langle\mathrm{id}\rangle$, since $\operatorname{Stab}_{\Gamma}(X \backslash O)$ is contained in $\operatorname{Stab}_{\Gamma}(\Gamma . x)$ which is itself trivial. This means that the labelling

$$
\phi(y)= \begin{cases}\phi_{1}(y) & \text { if } y \in O, \text { and } \\ \phi_{2}(y) & \text { if } y \in X \backslash O\end{cases}
$$

distinguishes $X$ under the action of $\Gamma$ with at most $k$ labels. If $O$ has at least two elements then the relation $|\Gamma|=\left|O \| \operatorname{Stab}_{\Gamma}\left(x^{\prime}\right)\right|$ shows that $\left|\operatorname{Stab}_{\Gamma}\left(x^{\prime}\right)\right|<n$ !. By Corollary 2.12 the set $X \backslash O$ can be distinguished under the action of $\operatorname{Stab}_{\Gamma}\left(x^{\prime}\right)$ with at most $k \leq n-1$ labels. This would mean that $X$ is $n$-1-distinguishable, which contradicts the hypothesis.

\section{Distinguishing numbers for $S_{4}$ actions}

Albertson and Collins showed that if a graph has automorphism group $S_{4}$ then its distinguishing number is either 2 or 4 (see [AC1]). We demonstrate here that the analogous statement for $S_{4}$-actions on sets is false even when restricted to faithful $S_{4}$-actions. This shows that the problem of distinguishing group actions is more general than the problem of distinguishing graphs.

The choice of $X$ with $D_{S_{4}}(X)=3$ was inspired by a conversation with Daniel Isaksen.

Theorem 3.1. If $S_{4}$ acts on $X$ then the distinguishing number $D_{S_{4}}(X)$ is either $1,2,3$, or 4 . If $S_{4}$ acts faithfully on $X$ then $D_{S_{4}}(X)$ is either 2,3 , or 4.

Proof. The distinguishing number of an $S_{4}$-action is $1,2,3$, or 4 by Corollary 2.12 .

The trivial $S_{4}$-action on the one-element set has distinguishing number 1 .

If $S_{4}$ acts on itself by translation, its distinguishing number is 2 by Proposition 2.2.

When $S_{4}$ acts on the 4-element set by all possible permutations its distinguishing number is 4 , by Theorem 2.15 .

Let $X$ be graph whose vertex set is the conjugacy class of the permutation (1234) and whose edge set consists of $\left(v, v^{\prime}\right)$ such that the permutation $v$ is the inverse of $v^{\prime}$. 
The graph $X$ is given in Figure 5, showing both a labelling $\phi$ indicated by the numbers within the vertices and the permutation corresponding to each vertex. Let $S_{4}$ act on $X$ by conjugation. (This action does consist of graph automorphisms since conjugation preserves inverses.) Figure 5 shows a 3-distinguishing labelling of $X$ under this action. The rest of this proof verifies that no 2-labelling $\phi^{\prime}$ distinguishes $X$ and so in fact $D_{S_{4}}(X)=3$.

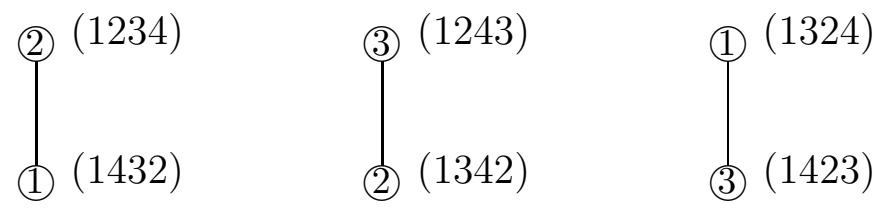

Figure 5: The graph $X$ with a 3-distinguishing labelling

Suppose $\phi^{\prime}$ gives both vertices of a component the same label for two different components, say without loss of generality that $\phi^{\prime}((1243))=\phi^{\prime}((1342))$ and $\phi^{\prime}((1324))=$ $\phi^{\prime}((1423))$. Then the action of (13)(24) on $X$ preserves each component, exchanging each of these pairs while fixing the first component, and so $\phi^{\prime}$ does not distinguish $X$. We assume $\phi^{\prime}$ does not label two components in this way.

Now suppose both vertices of one component share the same label, say without loss of generality $\phi^{\prime}((1234))=\phi^{\prime}((1432))$. The action of each of (13) and (24) exchanges the vertices (1234) and (1432) and exchanges the other two components, switching the components in the two possible ways. Thus $\phi^{\prime}$ cannot distinguish the graph.

Finally, suppose $\phi^{\prime}$ gives a different label to the two elements of each component, and say without loss of generality that $\phi^{\prime}((1234))=\phi^{\prime}((1243))=\phi^{\prime}((1324))$ and $\phi^{\prime}((1432))=$ $\phi^{\prime}((1342))=\phi^{\prime}((1423))$. Then the action of (14)(12) on $X$ cyclically permutes the three components while preserving the labelling, so $\phi^{\prime}$ does not distinguish $X$.

Albertson and Collins conjectured that if a graph $G$ has automorphism group $S_{n}$ and $D(G) \neq n$ then in fact $D(G) \leq \frac{n}{2}$ as long as $n$ is at least 4 . The previous theorem shows that this is false for general group actions. However, suppose the group action of $\Gamma$ on $X$ is faithful, namely $\operatorname{Stab}_{\Gamma}(X)=\langle i d\rangle$. For example, if $G$ is a graph then its automorphism group acts faithfully on $G$ because only the identity automorphism fixes each vertex.

We ask the following.

Question 3.2. Do there exist faithful group actions of $S_{n}$ on $X$ with $D_{S_{n}}(X)=n-1$ for arbitrarily large $n$ ?

\section{Distinguishing graphs using orbits}

In this section we apply the theory developed for distinguishing general group actions to graphs. Combining the general theory with properties of graphs that are invariant under automorphism, for instance distance or degree, permits these results to be extended. In Theorem 4.1 we distinguish trees. In Theorem 4.2 we describe all graphs with automorphism group $S_{n}$ and distinguishing number $n$. 
Cheng provided a different proof of Theorem 4.1 in [Ch, 2.2.4 and 2.2.5] as well as an algorithm to compute the distinguishing number of trees.

Theorem 4.1. If $T$ is a tree with maximum degree $d \geq 2$ then $D(T)$ is at most $d$. Otherwise $T$ is either a tree with one vertex and $D(T)=1$ or a tree with two vertices and $D(T)=2$.

Proof. The proof inducts on the number of vertex orbits in $T$. Suppose that $T$ consists of a single vertex orbit. Each graph automorphism preserves the degree of its vertices, in the sense that $\operatorname{deg}(v)=\operatorname{deg}(\sigma v)$ for each vertex $v$ and automorphism $\sigma$. Since all vertices in $T$ are in the same orbit, they must all be leaves. Consequently $T$ consists of either a single vertex or a single edge between two vertices, and $D(T)$ is as given.

Now assume $T$ has at least two orbits and let $d$ be the maximum degree of $T$. Let $O_{v}$ be any vertex orbit in $T$ which contains a leaf. This means that all of the vertices in $O_{v}$ are leaves. Write $T^{\prime}$ for the subgraph induced by $V(T)-O_{v}$. The graph $T^{\prime}$ is also a tree since no internal vertices were removed from $T$.

If $\sigma$ is a graph automorphism of $T$ then $\sigma O_{v}=O_{v}$ by definition. Let $\operatorname{Stab}\left(T^{\prime}\right)$ be the stabilizer of $T^{\prime}$ in $\operatorname{Aut}(T)$. Let $\sigma$ be in $\operatorname{Stab}\left(T^{\prime}\right)$ and choose an edge $u w$ from a vertex $u$ in $T^{\prime}$ to a vertex $w$ in $O_{v}$. The automorphism $\sigma$ sends the edge $u w$ to $(\sigma u)(\sigma w)=u(\sigma w)$. This means that the stabilizer of $T^{\prime}$ permutes vertices in $O_{v}$ with a shared neighbor.

Suppose $\phi$ is a distinguishing labelling of $T^{\prime}$. We extend $\phi$ to $T$ by requiring that for each vertex $u$ in $T^{\prime}$ that is adjacent to a vertex in $O_{v}$, the neighbors $N_{T}(u) \cap O_{v}$ are labelled distinctly. This requires at most $d$ labels, where $d$ is the maximum degree of $T$. By Lemma 2.3 the map $\phi$ is a $\max \left\{d, D\left(T^{\prime}\right)\right\}$-distinguishing of $T$. Since $T$ has at least two vertex orbits it has an internal vertex, so $d$ is at least 2 . The maximum degree of the subtree $T^{\prime}$ is no greater than $d$, and hence the result holds by induction on the number of vertex orbits.

The tree constructed by attaching $i$ leaves and $d-i$ paths of different lengths to a central root has distinguishing number $i$ and maximum degree $d$. Figure 6 shows such a tree when $i$ is 3 and $d$ is 5 . This shows that the bound in Theorem 4.1 is sharp.

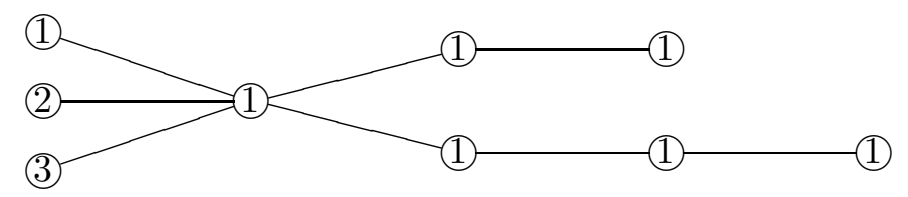

Figure 6: A tree with distinguishing number 3 and maximum degree 5

The next result characterizes graphs whose automorphism group is $S_{n}$ and whose distinguishing number is $n$. This proves a conjecture in [AC1].

Theorem 4.2. If $\operatorname{Aut}(G)=S_{n}$ and $D(G)=n$ then one orbit of $G$ is a copy of $K_{n}$ or $\overline{K_{n}}$ and the rest are 1 -orbits. 
Proof. Theorem 2.15 showed that $G$ has one vertex orbit $O$ with $n$ elements upon which $\operatorname{Aut}(G)$ acts as all possible permutations. All the other vertex orbits of $G$ are 1-orbits.

If $u$ and $v$ are two vertices in $O$ with an edge between them then $(\sigma u)(\sigma v)$ will be an edge for each $\sigma$ in $\operatorname{Aut}(G)$. Since the group of all permutations acts doubly transitively on the $n$ element set, there exists $\sigma$ with $\sigma u=u^{\prime}$ and $\sigma v=v^{\prime}$ for each pair of vertices $u^{\prime}, v^{\prime}$. If $O$ does not induce $\overline{K_{n}}$ then it induces $K_{n}$.

\section{References}

[AC1] M. Albertson and K. Collins, Symmetry Breaking in Graphs, Elect. J. Comb. 3 1996, \#R18.

[AC2] M. Albertson and K. Collins, An introduction to symmetry breaking in graphs, Graph Theory Notes of New York XXX 1996, 6-7.

[Co] K. Collins, personal communication.

[Ch] C. Cheng, Three problems in graph labelling, Ph. D. dissertation, The Johns Hopkins University, 1999.

[FH] W. Fulton and J. Harris, Representation Theory, Springer-Verlag, New York, 1991.

[L] S. Lang, Algebra, Addison Wesley, New York, 1993. 